\title{
Technical Publication Transfer Test with Vitro Corporation MIL-M-28001 (SGML) and MIL-D-28000 Class I (IGES)
}

\section{Quick Short Test Report}

\section{September 18, 1990}

\section{Prepared by}

Lawrence Livermore

National Laboratory

LLNL Contact

Jill Farrell

(415) 423-6348

SYSCON Contact

Jeff Howells

(619) 296-0085

AFLC Contact

Mel Lammers

(513) 257-3085

\section{Prepared for}

Air Force Logistics Command AITI Project 


\section{Contents}

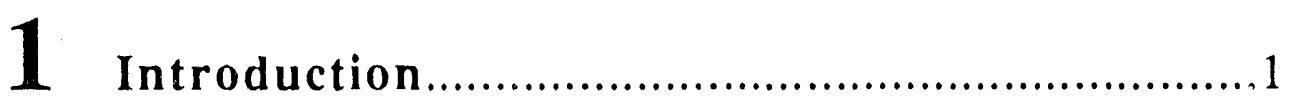

2. Test Parameters..............................................

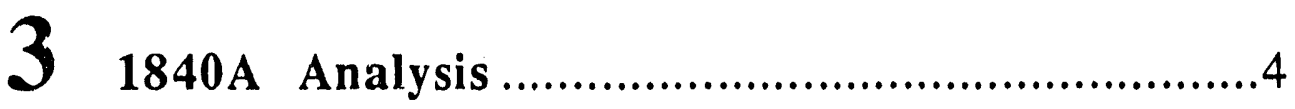

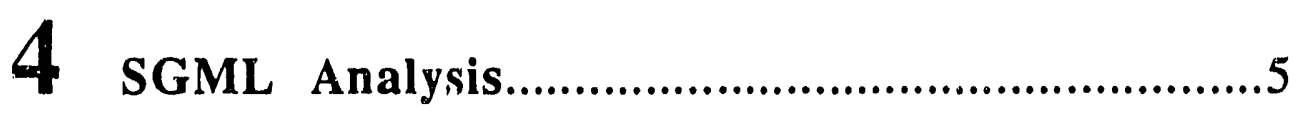

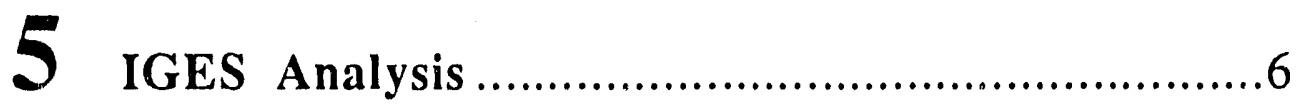

6 Conclusions and Recommendations..................... 


\section{Introduction}

\subsection{Background}

The DoD Computer-aided Acquisition and Logistic Support (CALS) Test Network (CTN) is conducting tests of the military standard for the Automated Interchange of Technical Information, MIL-STD-1840A, and its companion suite of military specifications. The CTN is a DoD-sponsored confederation of voluntary participants from industry and government managed by the Air Force Logistics Command.

The primary objective of the CTN is to evaluate the effectiveness of the CALS standards (Standards) for technical data interchange and to demonstrate the technical capabilities and operational suitability of those Standards. Two general categories of tests are performed to evaluate the Standards, formal and informal. Formal tests are large, comprehensive tests that follow a written test plan, require specific authorization from DoD, and may take months to prepare, execute, and report.

Informal tests are quick and short, taking only a few hours to set up and execute. They are used by the CTN technical staff to broaden the testing base by including representative samples of the many systems and applications used by CTN participants. They also allow the CTN staff to gain feedback from many industry and government interpretations of the Standards, to increase the base of participation in the CALS initiative, and to respond, in a timely manner, to the many requests for help that come from participants. Participants take part voluntarily and are benefited by receiving an evaluation oi their latest implementation (interpretation) of the Standards, interacting with the CTN technical staff, gaining experience in use of the Standards, and developing increased confidence in them. The results of informal tests are reported in Quick Short Test Reports (QSTRs) that briefly summarize the standard(s) tested, the hardware and software used, the nature of the test, and the results.

\subsection{Purpose}

The purpose of the informal test reported in this QSTR was to analyze Vitro Corporation's interpretation and use of the CALS standards in transferring technical publications data. Vitro used its CALS Technical Data Interchange System to produce data in accordance with the Standards and delivered it to the CTN technical staff on a 9-track magnetic tape. 


\section{Test Parameters}

Test Plan:

CTN89-TM-04

Date of

Evaluation:

August 28, 1989

Evaluators:

Lawrence Livermore National Laboratory

P.O. Box 808, L-542

Livermore, CA 94550

SYSCON Corporation

3990 Sherman Street

San Diego, CA 92110

Data

Originator:

Vitro Corporation

CALS Information Systems

14000 Georgia Avenue

Silver Spring, MD 20906-2972

Data

Description:

Army Maintenance Manual TM-11-5845-201-12 entitled "Operators and Organizational Maintenance Manual Sonar Sounding Set AN/SNQ-21"

1 document declaration file

1 text file

5 IGES files

Data

Source System: Vitro CALS Technical Data Interchange System incorporating:

1840A Compugraphic CAPS CALS for tape generation

SGML Compugraphic QUADEX for original text creation

Shaffstall System 6000 for conversion to SGML

Softquad SGMLEdit and Compugraphics CAPS CALS for SGML parsing and editing

IGES GTX DCAD for scanning and vector editing

GTX IGESCNV for IGES translation

Rosetta Technologies PrEDITOR and PreVIEW for IGES analysis and editing 
Evaluation

Tools Uśed:

1840A

SGML

IGES

Standards

Tested:
CTN TAPEVAL

Datalogics, Softquad, and Software Exoterica Instance Parsers

IGES Data Analysis Inc. Parser/Verifier

Glatz Ass. IGES Model Testing System

Rosetta Technologies, Inc. Pre VIEW

MIL-STD-1840A Notice 1 (1840A)

MIL-M-28001 (28001)

MIL-D-28000 Amendment 1 (28000) Class I 


\section{1840A Analysis}

\subsection{External Packaging}

The Vitro packaging conformed to the requirements. Overall, the entire package and supporting documentation was of the highest quality.

\subsection{Transmission Envelope}

\subsubsection{Tape Formats}

All the files on the tape had the correct record formats, record lengths, and block sizes.

\subsubsection{Declaration Files and Header Fields}

The CTN's TAPEVAL log file was analyzed for 1840A header errors. No errors were discovered in the $1840 \mathrm{~A}$ declaration files or header fields. 
CTN Test Report 90-013

September 18,1990

\section{SGML Analysis}

A brief analysis was performed on the SGML text file using three different instance parser software packages. The Datalogics Instance Parser indicated the following problems with the instance. The very first tag, " $<$ doc", had no end angle-bracket. Since this may have been a typographical error, the end angle-bracket was added. The parser claimed that a "<para0>" that follows two consecutive tables contained in a "<para0 $>$ " is illegal. The parser also claimed tíat "<paratext $>$ " may not contain a "<ftnote>". The Datalogics Parser could not continue after encountering this problem. It is clear in the 28001 DTD that "<paratext $>$ " may contain a "<ftnote $>$ " via the "\%text" entity.

The Software Exoterica instance parse yielded completely different results. The missing end angle-bracket after the "doc" tag was ignored. The parser flagged the footnotes tagged as "ft2", "ft 3 ", and " $\mathrm{ft} 4$ " as unexpected text contained in a "paratext" tag. According to 28001 , zero or more footnotes are allowed at the end of the paratext content model (see the \%text; entity).

The Softquad Instance Parser indicated no errors in the SGML markup. It did ignore the missing end angle-bracket. It appears as though the tables in this SGML text file were normalized. They contain end-row tags and entry tags with no content. Both sets of these tags are optional. The errors encountered using the Datalogics and Software Exoterica parsers appear to be content model interpretation problems. None of the parsers used indicated serious SGML errors except for the missing end angle-bracket caught by the Datalogics Instance Parser.

The development materials sent with the tape included a section that made references to parsing problems. These problems mentioned were encountered as the document was prepared for the transmission. The hard copy document sent with the materials was the original instance. 


\section{IGES Analysis}

Analysis of the five IGES files showed that they correctly represented the graphics of Vitro's illustrations and contained proper Start Sections, however, they did not conform to 28000 Class I in all respects. The areas of non-conformance were:

1. Drawing, Drawing Name, and View Entities that are required by 28000 were missing.

2. All entities were not on layer zero as required by Class I.

3. The text was written in a text font not allowed by 28000 .

4. Several Subordinate, Use, and other Directory Entry settings were in minor non-conformance to the IGES Specification. 


\section{Conclusions and Recommendations}

Technically, the 1840A transmission envelope of the Vitro tape was correct. Overall, the supporting documentation (copies of document declaration files, source data files, illustrations, and comments) was excellent and should serve as a model for an ideal transmission package of $1840 \mathrm{~A}$ data.

Except for the missing end angle-bracket mentioned, the SGML-coded text data appears to conform to 28001 . The vendors of the instance parse software packages should be notified of the different results obtained from each parse.

The IGES files were not in complete conformance with 28000 Class I, yet the graphics they contained perfectly represented Vitro's original illustrations. 

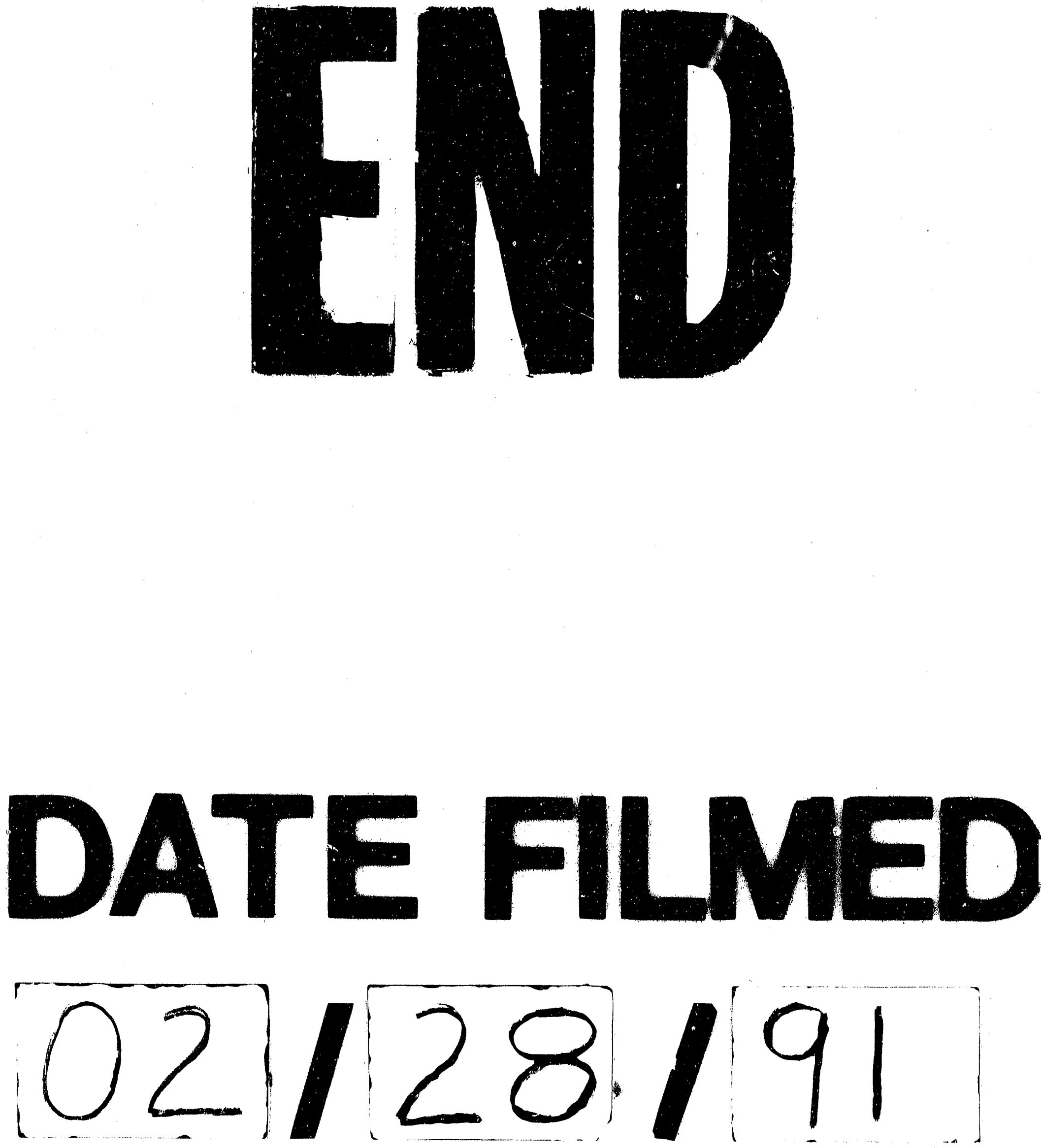
